\title{
Resultados del Programa Nacional de Implantes Cocleares y Garantías Explícitas en Salud en beneficiarios pertenecientes al Servicio de Salud Aconcagua
}

\section{Results of the national program of cochlear implants and explicit health guarantees in beneficiaries belonging to the Aconcagua health service}

\author{
Cristián Guzmán O. ${ }^{1,2}$, Eduardo Fuentes-López ${ }^{3}$, Felipe Cardemil M. ${ }^{4,5}$
}

\begin{abstract}
Resumen
Introducción: En Chile, desde la ejecución de políticas públicas vinculadas a la implementación de implantes cocleares, sólo existen estudios elaborados y publicados en la Región Metropolitana que han buscado describir los resultados que estas han alcanzado en la población. Objetivo: Describir los resultados del Programa Nacional de Implantes Cocleares y Garantías Explícitas en Salud en pacientes beneficiados pertenecientes al Servicio de Salud Aconcagua. Material y Método: Estudio de corte transversal. Se reportan los resultados de la totalidad de pacientes beneficiados implantados en el período 20032016 del Servicio de Salud Aconcagua. Resultados: Del total de personas postuladas a nivel nacional, el 3\% $(n=17)$ fueron postuladas desde el Servicio de Salud Aconcagua, de las cuales, el 58,8\% $(\mathrm{n}=10)$, fueron beneficiadas con implante coclear. La totalidad de los beneficiados fueron pesquisados a través de consulta en policlínico de Otorrinolaringología. La mediana de diagnóstico auditivo fue 22 meses (Q1-Q3:16-24), implementación de audífonos 24 meses (Q1-Q3:17-25), implementación de implante coclear 50 meses (Q1-Q3:29-56) y encendido de 52 (Q1-Q3:33-57). La mediana de ganancia funcional con implante fue 58,4 dB (Q1-Q3:46-65); estadísticamente significativa $(\mathrm{p}<0,05)$. Conclusión: Los resultados en pacientes usuarios de implante coclear son satisfactorios. Es necesario ampliar la cobertura de estas políticas en el valle del Aconcagua, en coherencia con la mejora de procesos vinculados al diagnóstico oportuno de hipoacusia y posterior implementación de ayudas auditivas en tiempos que la evidencia sugiere pertinentes. Palabras clave: hipoacusia, implante coclear, políticas públicas en salud.
\end{abstract}

\begin{abstract}
Introduction: In Chile, since the implementation of public policies linked to the program of cochlear implants, the only published studies were prepared in the Metropolitan Region, that have sought to describe the results that these responses have obtained in the population. Aim: Describe the results of the National Cochlear Implants Program and explicit health guarantees in beneficiary patients belonging to Aconcagua health service. Material and Method: Cross-sectional study. The results of all the patients benefited implanted in the period 2003-2016 are reported. Results: Of the total number of people nominated nationwide, $3 \%(n=17)$ were chosen from the Aconcagua health service, of which $58.8 \%(n=10)$ were benefited with cochlear implants. All the benefited patients were investigated through consultation in the Otolaryngology polyclinic. The median auditory diagnosis was 22 months (Q1-Q3: 16-24), hearing aid implementation 24 months (Q1-Q3: 17-25), cochlear implant implementation 50-month (Q1-Q3: 29-56) and on of cochlear implant 52 months (Q1-Q3: 33-57). The median functional gain with implant was $58.4 \mathrm{~dB}$ (Q1-Q3: 46-65); statistically significant $(p<0.05)$. Conclusion: The results in patients using cochlear implants are satisfactory. It is necessary to expand the coverage of these public policies in the Aconcagua valley, consistent with the improvement of the processes related to the timely diagnosis of hearing loss and subsequent implementation of hearing aids in the times that the relevant evidence.
\end{abstract}

Keywords: hearing loss, cochlear implant, public health policies.
'Escuela de Fonoaudiología, Facultad de Medicina, Universidad de Valparaíso. San Felipe, Chile. 2Unidad de Otorrinolaringología, Hospital San Camilo. San Felipe, Chile. ${ }^{3}$ Fonoaudiología, Departamento de Ciencias de la Salud, Facultad de Medicina, Pontificia Universidad Católica de Chile. Santiago, Chile. ${ }^{4}$ Departamento de Otorrinolaringología, Facultad de Medicina, Universidad de Chile. Santiago, Chile. ${ }^{5}$ Departamento de Otorrinolaringología, Clínica Las Condes. Santiago, Chile.

Los autores declaran no tener conflictos de interés.

Recibido el 26 de septiembre de 2019. Aceptado el 24 de abril de 2020.

Correspondencia: Cristián Guzmán O. Escuela de Fonoaudiología, Facultad de Medicina, Universidad de Valparaíso, Camino la Troya esquina El Convento. San Felipe, Chile. Email: cristian.guzman@uv.cl 


\section{Introducción}

Actualmente, se estima que el 5,3\% de la población mundial padece algún grado de hipoacusia $(\mathrm{HA})^{1}$. En Chile, la HA constituye el 8,74\% de las deficiencias causales de discapacidad $^{2}$ y es la tercera causa de discapacidad infantil, con una incidencia de 1 a 3 por cada 1.000 recién nacidos vivos y 8 por cada 1.000 en menores con antecedentes de riesgo ${ }^{3}$. En adultos, la prevalencia de HA en mayores de 65 años es de un 52\%, mientras que, un 31,4\% de los adultos mayores de 18 años refieren experimentar discapacidad asociada a problemas auditivos ${ }^{4}$.

La HA afecta la calidad de vida y el bienestar de quienes la padecen debido a que no sólo compromete el desarrollo comunicativo, sino que también, compromete el desarrollo de aspectos cognitivos, educativos, psicológicos y sociales ${ }^{5,6}$. Hace algo más de una década, las opciones terapéuticas para la población con diagnóstico de HA sólo sugerían el uso de audífonos convencionales, ingreso a escuelas diferenciales y/o terapias educacionales. Sin embargo, en algunos casos, cuando las pérdidas de audición son severas a profundas en términos del compromiso funcional, estas estrategias no son suficientes para lograr niveles eficientes de audición de acuerdo con las necesidades de cada individuo ${ }^{7}$. Este es el caso de pacientes con diagnóstico de hipoacusia sensorioneural (HSN) severa o profunda, que con el uso de audífonos ven limitados los beneficios de amplificación del sonido restringiendo con ello el desarrollo del lenguaje ${ }^{8}$. En este contexto, una intervención costo-efectiva para el tratamiento de HSN severas a profundas es la implementación auditiva a través del implante coclear (IC) ${ }^{9}$. Existe consenso generalizado sobre el beneficio del IC en todas las etapas del desarrollo, sobre todo cuando es posible acceder tempranamente al dispositivo ${ }^{10}$. Sin embargo, los beneficios también dependen de variables como: edad de aparición de pérdida auditiva, tiempo de privación auditiva, plasticidad neural y edad en que se realiza la implantación del dispositivo ${ }^{7}$. Por otro lado, existen covariables como patologías asociadas, desarrollo psicomotor y otras de índole social (nivel socioeconómico y apoyo de terapia de habilitación/rehabilitación pre y postimplan- te) que también influyen sobre los beneficios asociados al uso del $\mathrm{IC}^{11}$.

En Chile, la implantación coclear comenzó en la década de los 90, desde la prestación privada de servicios, específicamente a través del Programa de Implante Coclear de la Clínica Las Condes, el año $1993^{12}$. Desde la modalidad pública institucional, el año 2003 se creó el Programa Nacional de Implantes Cocleares (PNIC), financiado por el Fondo Nacional de Salud (FONASA) ${ }^{13}$, y luego, como resultado de las Garantías Explícitas en Salud (GES), se incorporaron gradualmente a la red pública de prestaciones la implementación de IC a través de dos problemas de salud específicos; hipoacusia neurosensorial bilateral del prematuro $(2015)^{14}$ y tratamiento de hipoacusia moderada en menores de 2 años $(2013)^{3}$.

\section{Objetivo}

El objetivo del presente estudio es describir los resultados del Programa Nacional de Implantes Cocleares y Garantías Explícitas en Salud en pacientes beneficiados pertenecientes al Servicio de Salud Aconcagua entre los años 2003-2016.

\section{Material y Método}

Se realizó un estudio de corte transversal que incluyó la totalidad de pacientes implantados por el PNIC o bien por GES pertenecientes al Servicio de Salud Aconcagua (SSA) entre los años 2003 y 2016. El estudio contó con aprobación del Comité de Bioética del SSA (39/2017). Para dar cumplimiento al objetivo, se recopiló información a través de registros propios del Ministerio de Salud, Servicio de Salud Aconcagua y Unidad de Otorrinolaringología del Hospital San Camilo, además, de la realización de audiometrías a campo libre en pacientes beneficiados. Se recolectaron datos vinculados con la cantidad de pacientes beneficiados, antecedentes sociodemográficos (sexo, edad, escolaridad y comuna de residencia) y antecedentes audiológicos relevantes (modalidad de pesquisa de HA, caracterización de HA, centro implantador, edad de diagnóstico auditivo, edad de implementación de audífonos, edad 
de implementación de implante y niveles de percepción acústica del habla mediante escala de Geers y Moog).

Los datos fueron analizados con el programa STATA 12. Para variables cuantitativas se confeccionaron tablas de frecuencia y calcularon medidas de tendencia central según correspondiera (media, mediana, desviación estándar y rango intercuartil (RIC: Q1-Q3). Para variables cualitativas: proporciones y distribuciones frecuenciales. Para analizar diferencias entre variables cuantitativas se utilizó el test no paramétrico de los rangos con signos de Wilcoxon. Se consideró significativa un alfa menor a 0,05 .

\section{Resultados}

Entre los años 2003-2016, a nivel nacional, se postularon 545 personas para recibir IC, de las cuales, el $3 \%$, equivalente a 17 personas, fueron postuladas a través del SSA. Respecto de la cobertura alcanzada a nivel nacional, del total de personas postuladas, el $70 \%$, equivalente a 354 personas, fueron beneficiadas con IC. En el SSA, el 58,8\%, equivalente a 10 personas, fueron beneficiadas con IC. Considerando el total de pacientes beneficiados en el SSA, el 70\%, equivalente a 7 personas, obtuvo el beneficio a través del PNIC, el 30\% restante, equivalente a 3 personas, obtuvo el beneficio a través de las GES para pacientes menores de 2 años portadores de HA. El 50\% de los beneficiados en el SS es de sexo femenino, la mediana de edad actual es de 8 (Q1-Q3: 5-11) años y la distribución de ellos según residencia en las comunas de la provincia del Aconcagua es homogénea (Tabla 1).

Del total de pacientes beneficiados en el SSA, el 30\% fue implantado en la Región Metropolitana, mientras que, el $70 \%$ restante, fue implantado en la $\mathrm{V}$ región. En este contexto, se implementaron 6 personas $(60 \%)$ en el Hospital Naval; 3 personas (30\%) en el Hospital Barros Luco Trudeau y una persona (10\%), en el Hospital Carlos Van Büren. La totalidad de los pacientes beneficiados en el SSA fueron pesquisados a través de consulta en policlínico de Otorrinolaringología del Hospital San Camilo. En lo referente a la edad de diagnóstico auditivo e implementación inicial de audífo-

\begin{tabular}{lc}
$\begin{array}{l}\text { Tabla 1. Distribución de los beneficiados según } \\
\text { comuna de residencia }\end{array}$ \\
\hline Comuna de residencia & Número de beneficiados \\
\hline San Felipe & 2 \\
\hline Catemu & 2 \\
\hline Llay Llay & 1 \\
\hline Rinconada & 1 \\
\hline Calle Larga & 1 \\
\hline San Esteban & 2 \\
\hline Hijuelas & 1 \\
\hline
\end{tabular}

nos, la mediana de diagnóstico auditivo fue de 22 meses (Q1-Q3:16-24), mientras que, la mediana de implementación de audífonos fue de 24 meses (Q1-Q3:17-25). El tiempo promedio de espera reportado entre el diagnóstico auditivo y la implementación de audífonos fue de 1,6 meses. La mediana de implementación del implante coclear fue de 50 meses (Q1-Q3: 29-56) y la mediana de edad de encendido del dispositivo fue de 52 meses (Q1-Q3: 33-57). El tiempo promedio de espera reportado entre la implementación del implante y su encendido fue de 1,7 meses. El tiempo promedio de espera entre la implementación de audífonos y la implementación de implantes cocleares es de 48 meses (Figura 1).

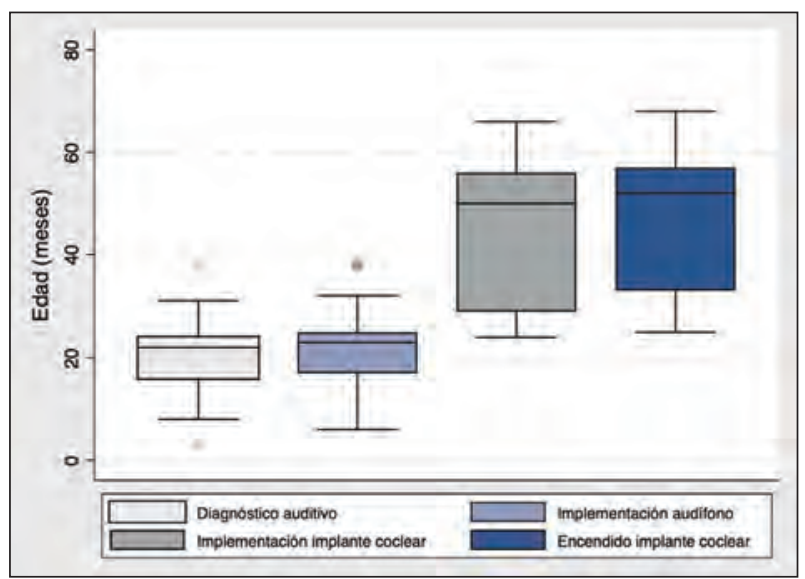

Figura 1. Comparación de edades de diagnóstico auditivo, implementación de ayudas auditivas y encendido de implante coclear. 


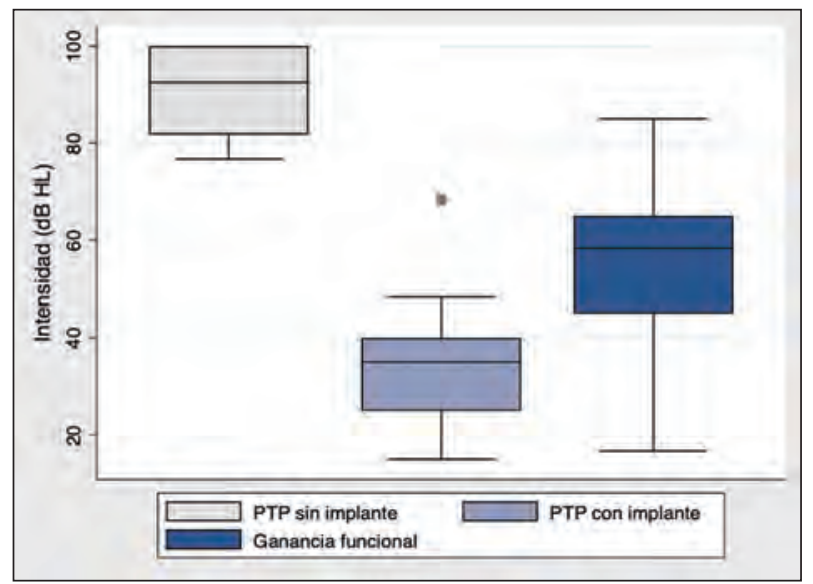

Figura 2. Comparación de promedios tonales puros (PTP) mediante audiometría a campo libre: sin implante, con implante y niveles de ganancia funcional.

En relación con la audiometría de campo libre y ganancia funcional, la mediana de promedios tonales puros obtenida sin implante coclear fue 92,5 dB (Q1-Q3: 81,7-100), mientras que, la mediana de promedios tonales puros obtenida con implante coclear encendido fue $35 \mathrm{~dB}$ (Q1-Q3: 25-40). Según lo anterior, los niveles de ganancia funcional evidenciados presentaron una mediana de 58,4 dB (Q1-Q3: 45-65), siendo esta ganancia estadísticamente significativa $(\mathrm{p}<0,05)$ entre las respuestas obtenidas (Figura 2). Al evaluar percepción acústica del habla según la escala de Geers \& Moog, se encontró una mediana de categoría 4 (Q1-Q3: 2-5). Actualmente, del total de pacientes beneficiados, 8 personas, equivalentes al $80 \%$, utiliza el IC, mientras que, 2 personas, equivalentes al $20 \%$, no lo utilizan.

\section{Discusión}

En Chile, existe escasa evidencia publicada que describa la cantidad de implantes cocleares que han sido entregados a la fecha a través del PNIC o bien por medio de GES. La evidencia señala que el IC es la alternativa de tratamiento que mejores resultados demuestra en variables vinculadas con la costo-efectividad, desarrollo del lenguaje, habilidades comunicativas, integración social y mejoras en la calidad de vida ${ }^{15,16}$. Sin embargo, en
Chile, existen barreras que determinan que, de un total de 545 personas postuladas entre los años 2003 y 2016, sólo 354 personas (70\%) reciban este beneficio. En el Servicio de Salud Aconcagua, en el mismo periodo de tiempo, 17 personas fueron postuladas, de las cuales, 10 personas $(58,8 \%)$ fueron beneficiadas con el IC. En este contexto, es necesario discutir esta realidad mediante el análisis de distintos factores que encuentran posiblemente un denominador común: los recursos disponibles; que se traducen en cantidad limitada de vacantes, centralización de postulaciones, recursos humanos e infraestructura escasa, y falta de métodos de pesquisa auditiva universales. Futuros estudios deberían abordar las variables mencionadas.

Respecto de la cantidad limitada de vacantes, entre los años 2003 y 2010, a través del PNIC, sólo era posible acceder a 10 dispositivos anuales concursables para todos los Servicios del país. Esto resultó no ser coherente con la demanda creciente de implantes y la oferta disponible, reduciendo por ello la cantidad de personas beneficiadas. No obstante, con la incorporación gradual de políticas públicas vinculadas a la implementación de IC al alero del programa GES; hipoacusia neurosensorial bilateral del prematuro $(2005)^{14} y$ tratamiento de hipoacusia moderada en menores de dos años $(2013)^{3}$, y al aumento de recursos inyectados al PNIC, es que la cantidad de pacientes beneficiados ha crecido con el pasar de los años, sin llegar por cierto a cubrir la totalidad de personas que lo requieren. En cuanto a la centralización de postulaciones y recursos profesionales e infraestructurales escasos, este trabajo reveló que sólo el 3\% del total de pacientes postulados a nivel nacional, entre los años 2003 y 2016, provino del Servicio de Salud Aconcagua, hallazgo coherente con reporte emanado del Departamento de Discapacidad y Rehabilitación del Ministerio de Salud que estableció que entre los años 2007 y 2010 , del total de personas postuladas, el $70 \%$ fueron procedentes de servicios de salud adscritos a la región Metropolitana y quinta región costa ${ }^{13}$.

En lo que respecta a los métodos de pesquisa auditiva universal, si bien estos no fueron sujetos de estudio en esta investigación, están en directa relación con lo que suceda en 
materia de cobertura de los IC. La experiencia internacional de programas de screening auditivo universal, demuestra que la detección temprana de HA es posible y que el diagnóstico acompañado de tratamiento audioprotésico e intervención fonoaudiológica permiten que los menores accedan a lenguaje oral en etapas tempranas y con ello el consiguiente desarrollo social, educativo y laboral ${ }^{17,18}$. Sin embargo, la cantidad de pacientes beneficiados con IC es menor a la cantidad de pacientes proyectados que debiesen cumplir con los criterios de implementación ${ }^{19}$. En Chile, si bien no existen cifras certeras que determinen la prevalencia de HA en la población, es posible proyectar que la cantidad de pacientes portadores de HA es mayor a la cantidad de pacientes beneficiados con implante entre los años 2003-2016. La anterior hipótesis se basa en investigaciones extranjeras que describen la prevalencia de HA cercana a 3 por cada 1.000 recién nacidos vivos y de 8 por cada 1.000 en recién nacidos vivos que presenten factores de riesgo asociados ${ }^{20,21}$.

Analizando la distribución de los beneficiados pertenecientes al Servicio de Salud Aconcagua según acceso al implante y relacionando esta información con lo descrito anteriormente, el 70\% de los pacientes beneficiados con IC lo hizo a través del PNIC y el $30 \%$ restante a través de la GES tratamiento de hipoacusia moderada en menores de dos años. La totalidad de los pacientes beneficiados fueron pesquisados mediante consulta en policlínico de Otorrinolaringología por no existir un programa de screening auditivo universal protocolizado en el servicio de salud $^{22,23}$. El año 2005 y 2013, con la incorporación al programa GES de las patologías auditivas asociadas a pacientes prematuros con factores de riesgo y consiguientemente, para pacientes menores de dos años, se logró avanzar en materias vinculadas con el tratamiento de dicha población. Sin embargo, la ejecución de estas políticas, no garantizan la implementación de programas de screening auditivo universales que permitan pesquisar a todos quienes poseen algún grado de HA y de esta forma brindar el tratamiento que en este momento se encuentra garantizado ${ }^{3,14}$.

Desde el año 2015, la Unidad de Otorrinolaringología del Hospital San Camilo, en alianza con la Escuela de Fonoaudiología de la Universidad de Valparaíso, está llevando a cabo la realización del programa de screening auditivo universal en el Servicio de Salud Aconcagua. En este contexto, el establecer alianzas estratégicas entre distintas instituciones relevantes de la sociedad ha resultado ser efectiva para resolver la carencia de programas de pesquisa auditiva ${ }^{9,24}$. En otras características audiológicas, esta investigación determinó que la mediana de edad de diagnóstico auditivo fue de 22 meses, mientras que, la mediana de edad de implementación de audífonos fue de 24 meses. Es necesario señalar que, si bien el tiempo de espera que existe entre el diagnóstico auditivo y la implementación de audífonos se acerca a los criterios establecidos por normativas tanto nacionales como internacionales ${ }^{14,25}$, la edad de diagnóstico auditivo está aumentada respecto de lo que propone la evidencia disponible; se sugiere que antes de los 6 meses de vida cronológica o corregida debe estar realizado el diagnóstico y la habilitación con ayudas auditivas en pro de potenciar el desarrollo integral de cada paciente como ha sido comentado anteriormente $^{26}$. En esta línea, pero en función de los datos vinculados a la edad de implantación y posterior encendido de los implantes, la mediana de edad de implementación del implante fue de 50 meses, mientras que, la mediana de edad de encendido del implante fue de 52 meses. Es preocupante analizar estas cifras, ya que si bien el tiempo promedio de espera que existe entre la implementación del implante y el posterior encendido está dentro de los estándares requeridos ${ }^{27}$, la edad de implementación de estos dispositivos está considerablemente por sobre las recomendaciones internacionales ${ }^{2,23,28}$.

Existe consenso respecto de la pertinencia que significa evaluar el desempeño de cada paciente mediante el uso de audífonos previa indicación de $\mathrm{IC}^{20}$, sin embargo, este tiempo de evaluación, no sobrepasa los 6 meses de uso. En el Servicio de Salud Aconcagua, el tiempo promedio que existe entre la implementación de audífonos y la posterior implementación de IC es de 26 meses, muy por sobre las recomendaciones entregadas por expertos ${ }^{14,29,30}$. Esta situación, es similar a lo reportado en un estudio previo desarrollado en la región 
Metropolitana, específicamente en el Hospital Barros Luco Trudeau, quienes en el año 2013 observaron una mediana de edad de implantación coclear de 48 meses 9 . En relación con los niveles de ganancia funcional evidenciados por los pacientes beneficiados, se obtuvo una mediana de $58,35 \mathrm{~dB}$, siendo este valor estadísticamente significativo. Este hallazgo es coherente con los datos publicados por el Servicio de Otorrinolaringología del Hospital Barros Luco Trudeau. Otro aspecto con resultados similares al estudio antes mencionado corresponde a los niveles de percepción acústica del habla evaluados mediante la escala de Geers \& Moog. Esta investigación determinó una mediana de categoría 4, cercana a la mediana de categoría 3 reportada por el Servicio de Otorrinolaringología del Hospital Barros Luco Trudeau?.

Considerando el total de pacientes beneficiados entre los años 2003 y 2016, actualmente, 8 pacientes utilizan su implante, mientras que, los dos restantes, no lo utilizan. Entre los motivos que determinan el no uso del implante, destaca la incomodidad que sugiere el uso del dispositivo, la falta de confortabilidad, escasa percepción de ganancia, sistema de escolarización incompatible, falta de motivación y escaso manejo de expectativas.

\section{Conclusión}

Los hallazgos de esta investigación describen los resultados obtenidos por el Programa Nacional de Implantes Cocleares y Garantías Explícitas en Salud en pacientes adscritos al Servicio de Salud Aconcagua. Considerando el número de pacientes beneficiados, es necesario ampliar la cobertura de estas políticas públicas en el Servicio de Salud Aconcagua, coherentemente con la mejora de procesos vinculados al diagnóstico oportuno de hipoacusia y posterior implementación de ayudas auditivas en los tiempos que la evidencia disponible lo sugiere.

\section{Agradecimientos}

Los autores desean reconocer el trabajo que realizan los profesionales de la Unidad de Otorrinolaringología del Hospital San Camilo vinculados a la implementación de las políticas públicas abordadas en este trabajo: Dres. Carmen Meléndez P., Cristian Morales S., Pamela Mena, Daniel Páez, Mario Álvarez, Álex León I.; Fonoaudiólogos Patricia Cabrea O., Nury González G., Nicolás Sabaj E.; Tecnólogo Médico Geovana Estay F. y Técnico Paramédico Jeanete González.

\section{Bibliografía}

1. WHO global estimates on prevalence of hearing loss. Organizacion Mundial de la Salud, 2012.

2. Ministerio de Salud. Guía práctica clínica: Implante coclear. 2008;23.

3. MINSAL. Guía Clínica AUGE: Tratamiento de Hipoacusia Moderada en Menores de 2 Años; 2013.

4. SENADIS. II Estudio Nacional de La Discapacidad.; 2016. http://observatorio.ministeriodesarrollosocial. gob.cl/endisc/docs/Libro_Resultados_II_Estudio_ Nacional_de_la_Discapacidad.pdf. Accessed April 28, 2017.

5. Guimarães V de C, Barbosa MA. Prevalence of auditory changes in newborns in a teaching hospital. Int Arch Otorhinolaryngol 2012;16:179185. Disponible en https://doi.org/10.7162/S180997772012000200005.

6. Chia EM, Wang JJ, Rochtchina E, Cumming RR, Newall P, Mitchell P. Hearing impairment and health-related quality of life: The blue mountains hearing study. Ear Hear 2007;28:187-195. Disponible en https://doi.org/10.1097/AUD.0b013e31803126b6.

7. Peñaranda A, Mendieta JC, Perdomo JA, et al. Beneficios económicos del implante coclear para la hipoacusia sensorineural profunda. Rev Panam Salud Pública 2012;31:325-331. Disponible en https://doi. org/10.1590/S1020-49892012000400009.

8. O’Neill C, O’Donoghue GM, Archbold SM, Normand C. A cost-utility analysis of pediatric cochlear implantation. Laryngoscope 2000;110:156-160. Disponible en https://doi.org/10.1097/00005537200001000-00028.

9. Rahal Mena P, Muñoz D, Cárdenas R, Mansilla F, Cardemil FM. Resultados del Programa Nacional de Implantes Cocleares: Servicio de Otorrinolaringología del Hospital Barros Luco Trudeau. Rev Otorrinolaringol Cir Cabeza Cuello 2013;73:231-237.

10. Sánchez AJ, Monfort M. Niños con implantación coclear bilateral: Variación en los resultados. Rev Logop Foniatr y Audiol 2010;30:130-135. Disponible en https://doi.org/10.1016/S0214-4603(10)70160-1.

11. O’Neill C, Archbold SM, O’Donoghue GM, McAlister DA, Nikolopoulos TP. Indirect costs, cost-utility variations and the funding of paediatric cochlear implantation. Int J Pediatr Otorhinolaryngol 
2001;58:53-57. Disponible en https://doi.org/10.1016/ S0165-5876(00)00466-3.

12. Otorrinolaringología - Clínica Las Condes. https://www.clinicalascondes.cl/CENTROSY-ESPECIALIDADES/ Especialidades/ Otorrinolaringologia/Noticias/Te-puede-interesar/ Encuentro-de-pacientes-con-implante-coclear.aspx. Accessed May 21, 2017.

13. Vivanco J. Informe de Estado de Avance Programa de Implantes Cocleares FONASA/MINSAL 2007-2009. Santiago; 2010.

14. De Salud M, De S, Pública S. Guía Clínica 2010 Hipoacusia Neurosensorial Bilateral del Prematuro. 2010.

15. Ramos-Macías A, Borkoski-Barreiro S, FalcónGonzález J, Ramos de Miguel A. Implante Coclear. Estado Actual y Futuro Cochlear Implant. Current State and Future. Rev Med Clin Condes 2016;27:798807. Disponible en https://doi.org/10.1016/j. rmclc.2016.11.011.

16. Martins M, de Lima F, Santos R, Santos A, Barreto V, de Jesus E. Cochlear implants: our experience and literature review. Int Arch Otorhinolaryngol 2013;16:476-481. Disponible en https://doi. org/10.7162/S1809-97772012000400008.

17. Trinidad-Ramos G, de Aguilar VA, JaudenesCasaubón C, Núñez-Batalla F, Sequí-Canet JM. Recomendaciones de la Comisión para la Detección Precoz de la Hipoacusia (CODEPEH) para 2010. Acta Otorrinolaringol Esp 2010;61:69-77. Disponible en https://doi.org/10.1016/j.otorri.2009.09.008.

18. Wu CC, Hung CC, Lin SY, et al. Newborn genetic screening for hearing impairment: a preliminary study at a tertiary center. PLoS One 2011;6:e22314. Disponible en https://doi.org/10.1371/journal. pone.0022314.

19. Morettin M, Santos MJD dos, Stefanini MR, Antonio F de L, Bevilacqua MC, Cardoso MRA. Measures of quality of life in children with cochlear implant: systematic review. Braz J Otorhinolaryngol 2013;79:375-381. Disponible en https://doi. org/10.5935/1808-8694.20130066.

20. Holden-Pitt L, Albertorio J. Thirty years of the Annual Survey of Deaf and Hard-of-Hearing Children \& Youth: a glance over the decades. Am Ann Deaf 1998;143:72-76.
21. Díaz C, Goycoolea M, Cardemil F. Hipoacusia: trascendencia, incidencia y prevalencia. Rev Med Clin Condes 2016;27:731-739. Disponible en https://doi. org/10.1016/j.rmclc.2016.11.003.

22. Gerner De García B, Gaffney C, Chacón S, Gaffney M. Overview of newborn hearing screening activities in Latin America. Rev Panam Salud Publica 2011;29. http://www.scielosp.org/pdf/rpsp/v29n3/01.pdf. Accessed September 13, 2017.

23. Raine C. Cochlear implants in the United Kingdom: Awareness and utilization. Cochlear Implants Int 2013;14:S32-S37. Disponible en https://doi.org/10.11 79/1467010013Z.00000000077.

24. Albertz N, Cardemil F, Rahal M, Mansilla F, Cárdenas R, Zitko P. Universal screening program and early intervention (USPEI) in congenital bilateral sensorineural hearing loss in Chile. Rev Med Chile 2013;141:1057-63. Disponible en https://doi. org/10.4067/S0034-98872013000800013.

25. American Speech-Language-Hearing Association. Cochlear Implant Frequently Asked Questions. American Speech-Language-Hearing Association. http://www.asha.org/public/hearing/CochlearImplant-Frequently-Asked-Questions/. Accessed June $1,2017$.

26. Torrente M. Hipoacusia y sistema de Garantías Explícitas en Salud (GES) hearing loss and sistema de Garantías Explícitas en Salud. Rev Med Clin Condes 2016;27:740-4. Disponible en https://doi. org/10.1016/j.rmclc.2016.11.004.

27. American Speech-Language-Hearing Association. Cochlear Implants 2004. Disponible en https://doi. org/10.1044/policy.TR2004-00041.

28. le Roux T, Vinck B, Butler I, et al. Predictors of pediatric cochlear implantation outcomes in South Africa. Int J Pediatr Otorhinolaryngol 2016;84:6170. Disponible en https://doi.org/10.1016/j. ijporl.2016.02.025.

29. Maggs J, Ambler M, Hanvey K. Trends in cochlear implant candidacy in children. Paediatr Child Health (Oxford) 2017:1-5. Disponible en https://doi. org/10.1016/j.paed.2017.06.002.

30. Parisier SC, Elexiades G, Hoffman R, Madell JR. Cochlear implants: Obstacles limiting application. Int Congr Ser 2004;1273:255-258. Disponible en https:// doi.org/10.1016/j.ics.2004.07.008. 